\title{
NOTAS BREVES SOBRE LA HISTORIA DE LAS SOCIEDADES IBÉRICAS PARA LOS RECURSOS GENÉTICOS ANIMALES
}

\author{
BRIEF NOTES ON THE HISTORY OF THE IBERIAN SOCIETIES FOR THE ANIMAL \\ GENETIC RESOURCES
}

Fernández, M. ${ }^{1}$ y Cima, $M .^{2}$

\begin{abstract}
'Sociedad Española para los Recursos Genéticos Animales (SERGA). Departamento de Genética. Universidad de Córdoba. Córdoba. España.mfr_barra@yahoo.es

${ }^{2}$ Sección Asturias. Sociedad Española para los Recursos Genéticos Animales (SERGA).
\end{abstract}

\section{Palabras clave adicionales}

Razas autóctonas. Conservación animal. Divulgación.

\section{RESUMEN}

Los recursos zoogenéticos son esenciales en la adaptación a las condiciones socioeconómicas y ambientales cambiantes de cada zona, incluido el cambio climático. Son la materia prima del mejorador animal y se encuentran entre los insumos más esenciales del ganadero. Son fundamentales para la producción agrícola sostenible.

Precisamente con esta filosofía nace en la Sociedad Española para los Recursos Genéticos Animales (SERGA) y la Sociedad Portuguesa de Recursos Genéticos Animais (SPREGA). Ambas tienen como objetivos fundamentalmente el conocimiento, estudio, protección, conservación y promoción de los recursos genéticos animales en general y ganaderos en particular, existentes en España y Portugal.

\section{SUMMARY}

Zoogenetic resources are essential in the adaptation to the socio-economical and environmental conditions permanently in change in every area, including the climatic change. These are the primary material in animal improvement and they are found in between the essential input of farmers. They are fundamental for the sustainable agriculture production.

Precisely with this philosophy the Spanish Society on Animal Genetic Resources (SERGA) and the Portuguese Society on Animal Genetic

\author{
AdDITIONAL KEYWORDS \\ Autochtonous breeds. Animal conservation. \\ Publication.
}

Resources were born. Both have the main objectives the knowledge, the study, the protection, the conservation and the promotion of the animal genetic resources in general, and the farm animals genetic particularly, both existing in Spain and Portugal.

\section{8-2011, MÁS DE DOS DÉCADAS DEHISTORIA}

Esta Sociedad se funda el primero de julio de 1988, con la firma en la Universidad de Zaragoza de sus estatutos fundacionales. Los Profesores Sierra Alfranca y Rodero Franganillo, Catedráticos de las Facultades de Veterinaria de las Universidades de Zaragoza y Córdoba, junto al ilustre Investigador del INIA Dr. Orozco Piñán, tristemente desaparecido, lideran una iniciativa de muchos científicos y técnicos españoles fruto de la preocupación por el incierto futuro que en aquellos años se les preveía a nuestras razas autóctonas.

La SERGA pronto se integra en las corrientes internacionales en la materia, participando en la fundación de Rare Breeds International en Edimburgo durante el verano de 1990.

El prestigio alcanzado por la sociedad y 


\section{FERNÁNDEZY CIMA}

su gran implantación hizo que desde su fundación asesorara al Ministerio de Agricultura, Pesca y Alimentación en múltiples aspectos relacionados con las razas autóctonas, hasta el punto de mantener un representante en el Comité de Razas de Ganado de España, órgano responsable de la gestión y mantenimiento del Catálogo Oficial de Razas de Ganado de España; el ser el Punto Focal Nacional Español (19941999) dentro del Programa Global de la FAO para la conservación de los recursos genéticos animales, y responsabilizarse de la realización (2004) de un estudio técnico para la elaboración del Informe Nacional Español para la elaboración por parte de FAO del Primer Informe Mundial sobre la situación de los Recursos Genéticos Animales (First Report on the State of the World's Animal Genetic Resources SoWAnGR).

En 1992 la Sociedad para contribuir con la celebración del quinto centenario del descubrimiento organizó en la Universidad de Córdoba el World Meeting on Domestic Animal Breeds related to the Discovery of America, en el que participaron los mejores especialistas en Conservación de los recursos zoogenéticos de todo el mundo. Las actas del mismo fueron publicadas en la revista científica Archivos de Zootecnia y aun hoy constituyen unos contenidos ampliamente consultados.

(http://www.uco.es/organiza/servicios/ publica/az/php/az.php?idioma_global= $0 \&$ revista $=41 \&$ indice $=31$ ).

En 1997 la SERGA organiza su primer congreso nacional en la Universidad de Córdoba con la participación de más de cien delegados de toda España.

A este primer congreso le siguió el segundo celebrado en Mallorca en 1998 (diciembre) con la misma participación.

La Facultad de Veterinaria de Lugo fue la anfitriona del tercer congreso en 1999 (11-13 de noviembre), en el cual se entabló una importante relación con los colegas portugueses que trabajan en el mismo ámbito (de ahí que se celebrara en Lugo el primer congreso ibérico de recursos genéticos animales, CIRGA), los cuales fundaron la Sociedad Portuguesa para los Recursos Genéticos Animales (SPREGA), con lo que a partir del año 1999 comenzamos a hacer nuestros eventos de una manera conjunta, alternativamente en cada país. Los trabajos presentados en este congreso han sido publicados en Archivos de Zootecnia (Vol. 50. N 189190).

En el año 2000 se celebró en la Estación Zootécnica Nacional de Santarem (Portugal) el segundo congreso ibérico de recursos genéticos animales y cuarto congreso SERGA.

En los dos últimos eventos la participación subió a más de 200 participantes.

Los siguientes congresos pasaron a celebrarse cada dos años, siendo la Facultad de Veterinaria de Madrid la anfitriona en 2002 del V congreso SERGA y III congreso ibérico, disparándose la participación hasta más de 400 delegados.

El siguiente congreso (VI congreso SERGA y IV congreso ibérico) celebrado por alternancia en tierras portuguesas, fue organizado en la Escuela de Zootecnia de Ponte de Lima (Portugal) en 2004, con un éxito similar al de eventos anteriores.

El VII congreso SERGA y V congreso ibérico sobre recursos genéticos animales se celebró en la Isla de la Palma del 4 al 7 de octubre de 2006.

El VIII congreso SERGA y VI congreso ibérico tuvo lugar en la capital portuguesa, Lisboa, del 16 al 18 de septiembre de 2008.

El último Congreso Nacional de SERGA (IX) e Ibérico (VII), se ha celebrado en el Principado de Asturias, Gijón, del 16 al 18 de septiembre de 2010.

Podemos decir que SERGA dispone de un acervo de cientos de artículos científicos de gran calidad publicados en sus Libros de Actas, la mayoría disponibles para el lector en la web de la Revista Archivos de Zootecnia (http://www.uco.es/organiza/servicios/ publica/az). 
Del mismo modo ha participado en la edición de libros de gran interés como Biodiversidad Porcina Iberoamericana. Caracterización y uso Sustentable (Ed. J.V. Delgado) y la organización de eventos relevantes como el Symposium on Pig Biodiversity.

En octubre de 2009 se publicó el libro Guia de campo de las razas autóctonas españolas, fruto de varios años de trabajo y de recopilación de información, que constituye el mayor compendio esquemático de los recursos zoogenéticos españoles, abordando todas las especies y todos los status actuales, en el que han participado decenas de miembros de la Sociedad de toda España. Este libro ha sido dedicado in memoriam al Dr. Orozco Piñán, Presidente Honorifico de SERGA.

SERGA partió como una organización de vocación centralista, y por ello tuvo que modificar sus estatutos a finales de los noventa para adaptarse a la estructura administrativa de nuestro país, en el que las competencias ganaderas están completamente transferidas a las comunidades autónomas.

Desde ese momento SERGA se constituye en secciones autonómicas, que mantienen un alto nivel de autonomía, en especial aquellas que se han organizado y cuentan con reglamentos de funcionamiento interno aprobados por nuestra asamblea.

En la actualidad la Junta Directiva de la SERGA está formada en su totalidad por especialistas en la materia, y mantiene una representatividad regional muy importante.
A través de la web http://www.uco.es/ serga se pone nuestra Sociedad a disposición de todos los veterinarios españoles con inquietudes en la conservación de la importante biodiversidad de los animales domésticos con la que cuenta nuestro país, la más importante de Europa, para que integren sus esfuerzos a los que venimos realizando en la SERGA en las últimas décadas.

En Portugal, SPREGA también realiza esta labor a través de su página web http:/ /www.sprega.com.pt/.

Tanto SERGA como SPREGA han unido sus esfuerzos junto a la Red Iberoamericana sobre la conservación de la biodiversidad de los animales domésticos locales y para el desarrollo rural sustentable, la cual promociona sus actividades en la web http:// www.uco.es/conbiand/Bienvenida.html.

\section{AGRADECIMIENTOS}

El último Congreso Nacional de SERGA (IX) e Ibérico (VII) celebrado en el Principado de Asturias, Gijón, del 16 al 18 de septiembre de 2010, contó con gran participación de asistentes y una numerosa presentación de trabajos técnicos científicos. Todos estos se han recogido en este volumen gracias a la financiación del Instituto Nacional de Investigación y Tecnología Agraria y Alimentaria (INIA) del Ministerio de Ciencia e Innovación del Gobierno de España, a través de la acción complementaria $A C 2010$ 00027-00-00 Organización del VII Congreso Ibérico sobre Recursos Genéticos Animales. 